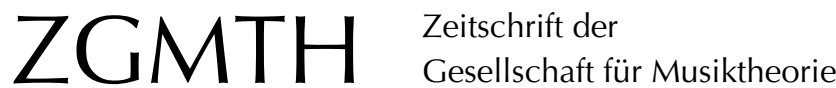

Lewandowski, Stephan (2010): `Fallende Quintanstiege. Ein Modellversuch. ZGMTH 7/1, 85-97. https://doi.org/10.31751/508

(C) 2010 Stephan Lewandowski

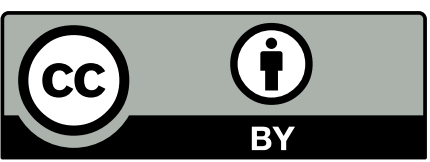

Dieser Text erscheint im Open Access und ist lizenziert unter einer Creative Commons Namensnennung 4.0 International Lizenz.

This is an open access article licensed under a

Creative Commons Attribution 4.0 International License.

veröffentlicht / first published: 01/02/2010

zuletzt geändert / last updated: 05/07/2011 


\title{
`Fallende Quintanstiege`
}

\section{Ein Modellversuch}

\author{
Stefan Lewandowski
}

Die Modellsystematik ist ein inzwischen weit verzweigtes Teilgebiet der musikalischen Analyse. Eine Vielzahl von neu- bzw. wiederentdeckten Kompositionslehren vor allem des 17.-19. Jahrhunderts in der zurückliegenden Zeit führt zu einer wachsenden und immer detaillierter erfassbaren Palette der zur Verfügung stehenden harmonisch-kontrapunktischen Modelle. Bislang wenig beachtet sind dabei ‘fallende Quintanstiege`, ein schrittweise abwärts verlaufendes Sequenzmodell, in welchem die Fundamentaltöne der zugrundeliegenden Harmonien innerhalb jedes Sequenzgliedes im Abstand einer steigenden Quinte/fallenden Quarte stehen. Verfolgen lassen sich sfallende Quintanstiege durch viele Epochen der Musikgeschichte hindurch, angefangen im 16. Jahrhundert bis hin zur Spätromantik. Auch in tonaler Gebrauchsmusik stellen sie ein nicht seltenes satztechnisches Modell dar. In verschiedenen kompositionsgeschichtlichen respektive stilistischen Kontexten nehmen sfallende Quintanstiege dabei unterschiedlichste semantische Bedeutungen an. So erscheint das Modell als Variante der Ausharmonisierung eines chromatischen Lamentobasses ebenso wie als satztechnisches wie hörend erfassbares Gegenstück zu aufsteigenden Quintfällen. Sein Auftreten in zahlreichen Musikbeispielen sowie sein Vorkommen in historischen Traktaten zur Kompositionslehre qualifizieren es als eigenständigen musikalischen Topos.

\section{Introduktion - Lamento}

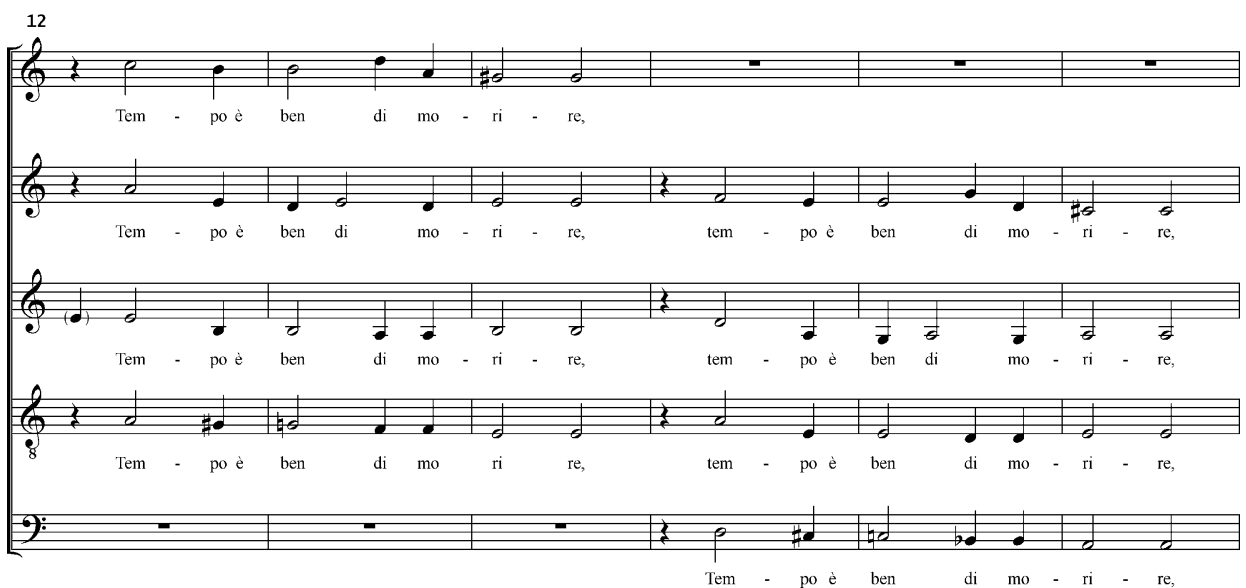

Beispiel 1: Carlo Gesualdo, Occhi del mio cor vita, 5. Madrigalbuch, Takte 12-17 
Die Beispiel 1 zeigt einen Ausschnitt aus dem Madrigal Occhi del mio cor vita von Carlo Gesualdo. "Tempo è ben di morire« heißt es im Text. Um dieser Stelle Nachdruck zu verleihen, bedient sich der Komponist ab Takt 15 des Mittels der Sequenzierung um eine Quinte tiefer. Das Partiturbild zeigt eine Abwärtsbewegung an: Zunächst pausiert der Bass, mit Beginn der Sequenz der erste Sopran. Schrittweise abwärts führt weiterhin die jeweils tiefste Stimme (beim ersten Mal der Tenor, dann der Bass). Das Rahmenintervall dieser Abwärtsbewegungen beträgt jeweils eine Quarte. Beide Sequenzierungen enden mit einem phrygischen Halbschluss.

Nichts von alledem ist überraschend - wie könnte eine solche Textstelle bei Gesualdo charakteristischer ausgedeutet sein als mit einem Lamento? Betrachtet man das Lamento jedoch genauer, so weist es einige Besonderheiten auf. Auffallend ist, dass die Bassbewegung hier gemischt chromatisch und diatonisch verläuft. Ein Halbtonschritt fehlt zwischen $g$ und $f$ sowie zwischen $c$ und $b$, um einen spassus duriusculus $z$ zu komplettieren. Genau an diesen Punkten erhält das Satzmodell ein besonderes Schmerzensmoment. Zum einen wird der zweite Sopran in Takt 13 sowie in Takt 16 auf den Zählzeiten 2-4 zur Patiensstimme, indem er eine sseptima syncopata serleidetı. Zum anderen - und dies ist klanglich weitaus entscheidender - enthält der zweite Sopran auf den ersten Zählzeiten besagter Takte jeweils die Quinte eines Dur-Akkords in Grundstellung, was eine maßgebliche Abweichung vom gewöhnlichen chromatischen Lamento-Modell bedeutet (Beispiel 2).

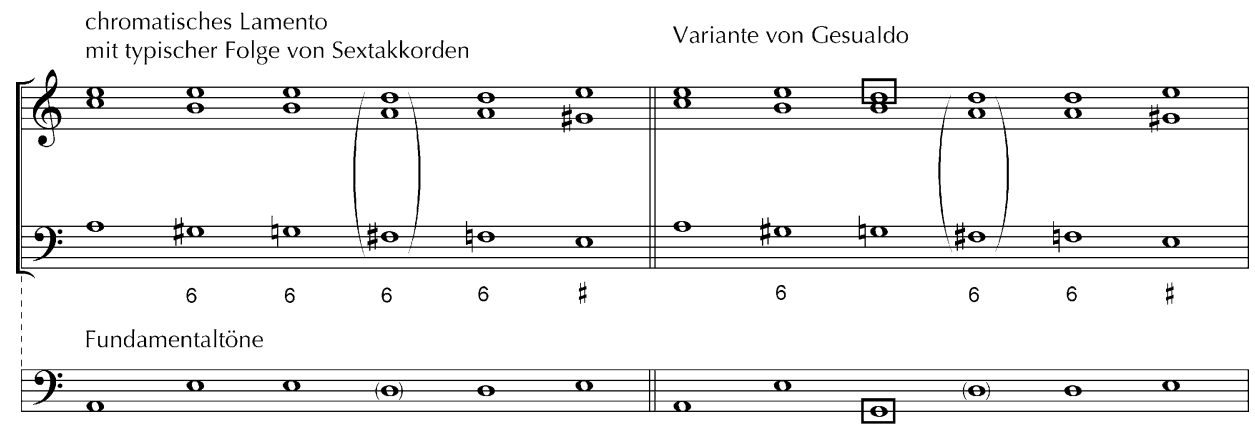

Beispiel 2: Harmonische Reduktionen von Lamento-Modellen mit Fundamentaltönen

Anstelle des Grundakkordes an dritter Position ist im chromatischen Lamento weitaus häufiger ein Sextakkord anzutreffen. Diese geringfügige Modifikation bedeutet eine klangliche und satztechnische Veränderung des zugrundeliegenden satztechnischen Modells Durch die spezielle Ausharmonisierung entsteht ein gesteigertes Maß an Expressivität. Darüber hinaus besitzt der Grundakkord einen neuen Fundamentalton ( $g$ statt e). Die Folge von Fundamentaltönen lässt sich beschreiben als absteigende Sequenzierung aufsteigender Quinten.

Ein Blick auf die unmittelbar anschließenden Takte aus Gesualdos Madrigal (Beispiel 3) zeigt eine dramaturgische Wende von einem absteigenden zu einem wieder ansteigenden Spannungsverlauf. Der Stimmungswandel geht einher mit dem Wechsel von 


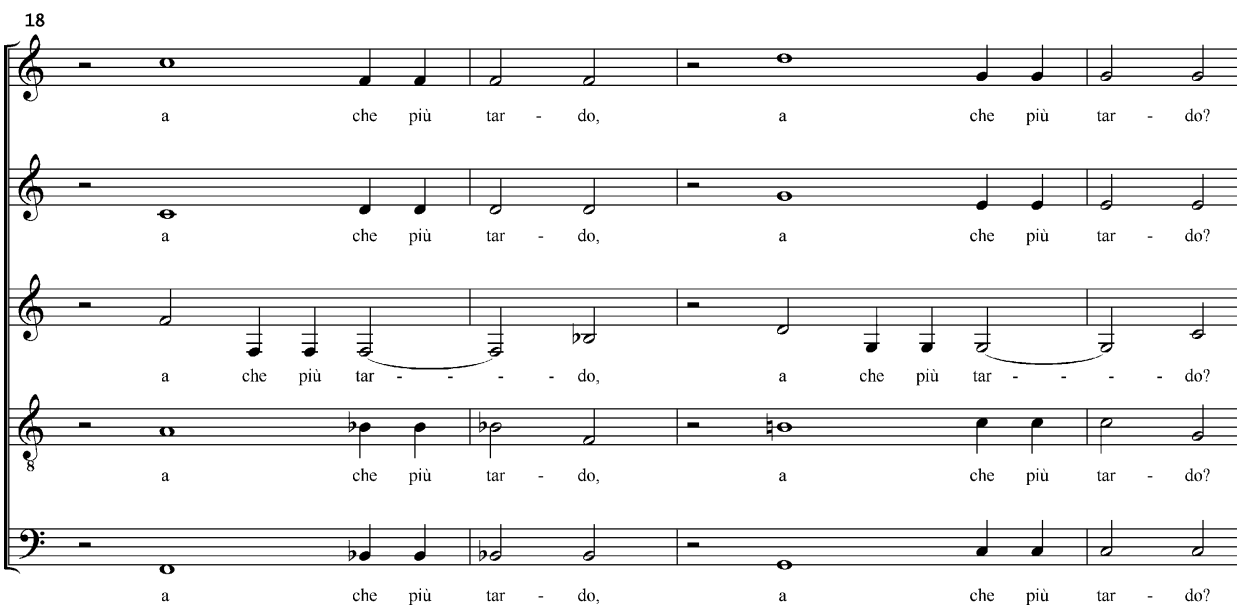

Beispiel 3: Carlo Gesualdo, Occhi del mio cor vita, 5. Madrigalbuch, T. 18-21

absteigenden zu aufsteigenden Sequenzierungen. Dies steht im Einklang mit den verwendeten satztechnischen Modellen: Nach dem Lamento folgen aufsteigende Quintfälle über der Frage »a che piú tardo?« (wozu später?). Mit dem Takt 17 lässt sich eine Grenze ziehen zwischen beiden Affekten bzw. harmonisch-kontrapunktischen Modellen; er bildet die vertikale Spiegelachse zwischen Lamento und aufsteigenden Quintfällen. Legt man beiden Modellen ihre Fundamentaltöne zugrunde, so ist ihre spiegelsymmetrische Beziehung zueinander erkennbar (Beispiel 4).

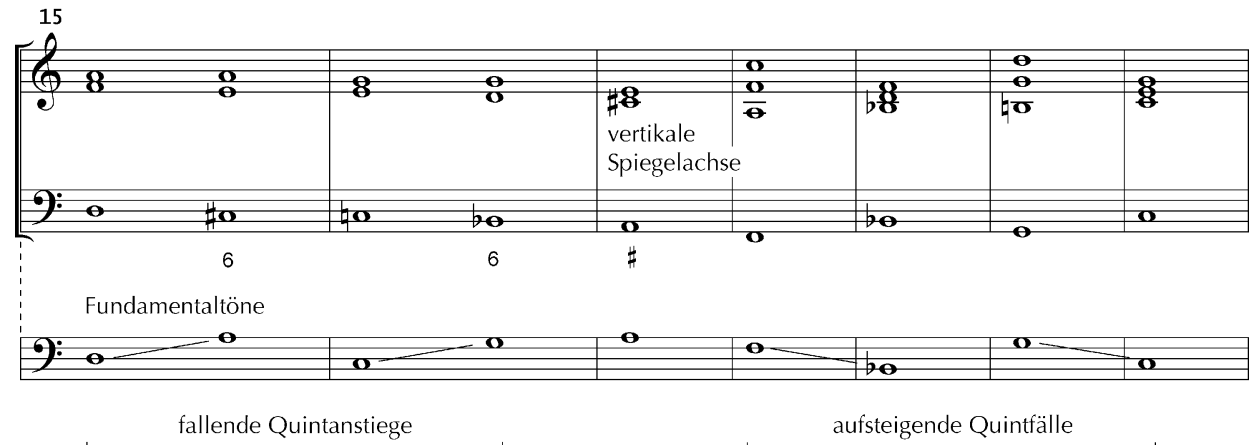

Beispiel 4: Lamento in Form fallender Quintanstiege als Spiegel aufsteigender Quintfälle (der Spiegel ist um einen Ganzton nach unten versetzt)

Fallende Quintanstiege lassen sich also nicht nur als Lamento-Variante auffassen, sondern ebenfalls als Spiegel (modulierender) aufsteigender Quintfälle. Dies emanzipiert das Modell als eigenständigen musikalischen Topos, zusammen mit der Tatsache, dass es mit wechselnder semantischer Bedeutung in der (dur-moll-tonalen) Musik des 16.-21. 
Jahrhunderts immer wiederkehrt. In den theoretischen Lehrwerken des 17.-19. Jahrhunderts ${ }^{1}$, in denen Modellsätze exemplifiziert werden, führen fallende Quintanstiege verglichen mit anderen Modellen nur selten ein Eigenleben zu eng scheint ihre Verwandtschaft mit dem Lamento zu sein. Gleiches gilt für die Lehrpraxis. ${ }^{2}$ Eine mögliche Ursache für das Fehlen fallender Quintanstiege in den theoretischen Lehrwerken ist der begrenzte epochale Rahmen, in dem das Modell vorkommt. In der Musikgeschichte klafft eine Lücke von zwei Jahrhunderten, in denen es so gut wie nicht anzutreffen ist. In der Spätrenaissance treten fallende Quintanstiege vor allem als Madrigalismen auf, in der Barockära und der Klassik sind sie seltener zu finden ${ }^{3}$, später erst wieder in der Romantik und ebenfalls in der Rock-/Popmusik der Gegenwart. Dies erscheint auch in gewisser Weise plausibel, lässt doch die Ausdruckskraft der Musiksprache des 19. Jahrhunderts durchaus Bezüge zur seconda prattica um 1600 erkennen.

\section{Thema mit Variationen - Fallende Quintanstiege in der Romantik}

Felix Mendelssohn-Bartholdys Sonate E-Dur für Klavier op. 6 ist ein Frühwerk des Komponisten. Bemerkenswert ist ihre formale Anlage: Sie besteht aus einem liedhaften Hauptsatz (Allegretto con espressione), einem Tempo di Minuetto, das eher an ein Scherzo erinnert, sowie einer rezitativischen Überleitung, die schließlich in einen Finalsatz (Molto allegro e vivace) mündet, der Elemente von Rondo und Sonatenhauptsatzform nach Beethovenschem Vorbild vereinigt. Alle Sätze gehen nahtlos ineinander über. Fantasieartig versponnen wirkt dieses Gebilde nicht zuletzt dadurch, dass das Hauptthema des ersten Satzes mehrfach wiederkehrt, so im Rezitativ und - mit abrundender Wirkung - am Schluss der Sonate. Nicht nur auf formaler Ebene experimentiert Mendelssohn, sondern auch mit harmonisch-kontrapunktischen Modellen. ${ }^{4}$ Die Experimentierfreudigkeit scheint sich dabei auszudrücken in der Verwendung seltenerer satztechnischer Modelle, wie im Tempo di Minuetto (Beispiel 5). Ab Takt 13 finden sich fallende Quintanstiege, die ausschließlich aus Grundakkorden bestehen. Das Modell beginnt eigentlich richtig in Takt 14, im Notentext mit dem Akzent gleichsam als `Startzeichen` markiert. Schon vor-

1 Zusammenstellungen modellbasierter Kompositionslehren finden sich u. a. bei Froebe 2006, 2007a und 2007b.

2 Das derzeit wohl profundeste und gleichzeitig für die Lehre am besten nutzbare Kompendium satztechnischer bzw. harmonisch-kontrapunktischer Modelle liefert Hartmut Fladt (2005a, vgl. auch 2005b). Lamento-Bässe werden auf S. 357 abgehandelt. Außerdem existiert bei Fladt die Kategorie »Quintstiege, mit anderen Intervallen kombiniert« (362). Die Kombinationsmöglichkeiten umfassen dabei die Versetzung der Quintanstiege um eine Terz aufwärts, um eine Terz abwärts (= Dur-MollParallelismus) sowie um eine Sekunde aufwärts. Nicht aufgelistet ist die Möglichkeit der Versetzung um eine Sekunde abwärts. Anknüpfend an die zeitgenössische Terminologie findet sich das Modell innerhalb einer eigenwilligen Klassifizierung bei Everard Sigal unter der Bezeichnung »steigende Quartschrittsequenz« (http://www.mu-sig.de/Theorie/Tonsatz/Tonsatz16.htm - 641c).

3 Eine Ausnahme stellt etwa Joseph Haydns vor 1766 entstandene Symphonie Nr. 16, B-Dur, Hob. I:16, dar, in deren Kopfsatz Haydn mehrfach fallende Quintanstiege verwendet. Das Modell bestimmt nicht nur die Harmonik der eröffnenden Takte 1-24, es bildet darüber hinaus die harmonische Grundlage weiter Strecken im Durchführungsteil.

4 Insbesondere der Schlusssatz ist diesbezüglich eine wahre Fundgrube. 
her könnte man, die Sinneinheit von fünf Takten berücksichtigend, den Beginn mit dem cis-Moll-Akkord in Takt 13 ansetzen. Das $h^{2}$ in der Oberstimme hat jedoch keinen Basston. Ein gedachtes gis als Fundamentalton würde zwar dem Schema fallender Quintanstiege gerecht, die Harmonie H-Dur auf der ersten Zählzeit von Takt 14 ergäbe allerdings einen mindestens ebenso logischen Anschluss. Der in Klammern stehende Fundamentalton dis im Takt 16 erklingt nicht mehr als Grundton in der Bassstimme sondern als Terz. Über diesen Sextakkord erfolgt der Ausstieg aus dem Modell, an das sich eine Kadenz nach H-Dur anschließt. Auffällig ist der Oktavkanon zwischen beiden Außenstimmen, der um einen Viertelschlag versetzt läuft. Die Mittelstimme stellt eine Austerzung der später einsetzenden Kanonstimme dar. Anhand dieser satztechnischen Faktur wird der Bezug fallender Quintanstiege zur Tradition des >contrapunto alla mente überdeutlich. ${ }^{5}$

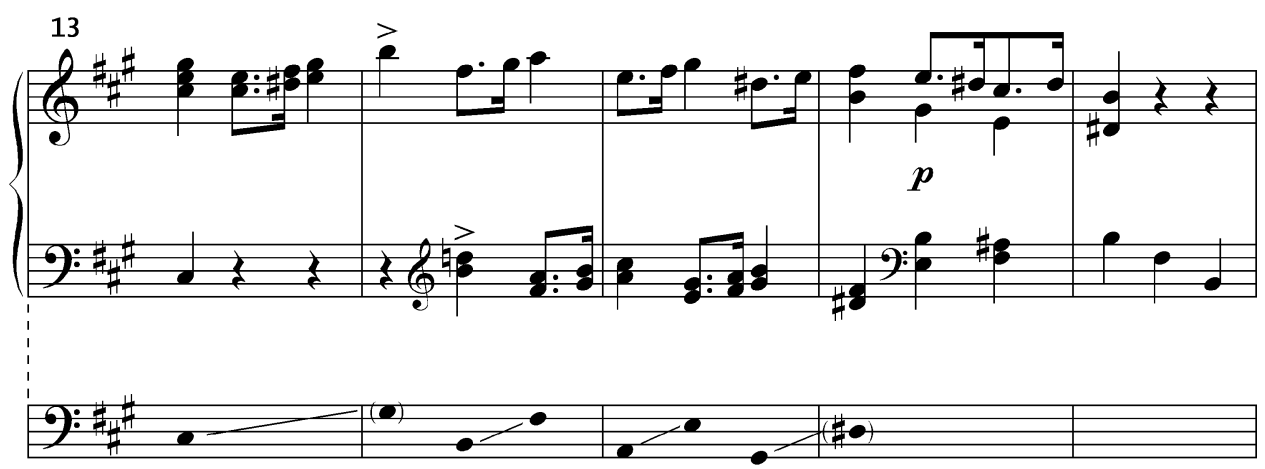

Beispiel 5: Felix Mendelssohn-Bartholdy: Sonate E-Dur für Klavier op. 6, Tempo di Minuetto, T. 13-17

Dem Modell ist eine unverkennbare Harmonik zu eigen. In Mendelssohns Klaviersonate verleiht sie nicht nur der Passage im zweiten Satz ihren besonderen Ausdruck sondern darüber hinaus auch dem Rondo-Thema des Finalsatzes seinen Wiedererkennungswert. Beispiel 6 zeigt den Ausschnitt des Themas, der eine komprimierte Fassung der fallenden Quintanstiege aus dem Tempo di Minuetto beinhaltet. Zugleich wird durch die satzübergreifende Wiederkehr des Modells der Grad an Geschlossenheit in Form und Harmonik im Werk noch erhöht.

Der erste Satz aus Schuberts G-Dur-Streichquartett (Beispiel 7) berührt den Hörer nach wenigen dramatischen, nahezu chaotisch hereinbrechenden Eröffnungstakten mit einer Pianissimo-Stelle mit surrenden Tremoli im Hintergrund. Dieser Passage ist ein Charakter des Religiös-Erhabenen eigen, der an Passagen später Beethoven-Streichquartette

5 Fallende Quintanstiege als harmonisches Gerüst für Kanonmodelle finden sich bei Ludovico Zacconi 1622, 189. Auch in die spätere Generalbasslehre haben sie als Satzmodell, zum Teil mit kanonischer Struktur, Eingang gefunden. Georg Muffat listet einige solcher Modelle auf, bei denen »der Baß quartweis herunter springt« (1699, 89); vgl. Froebe 2007b. 


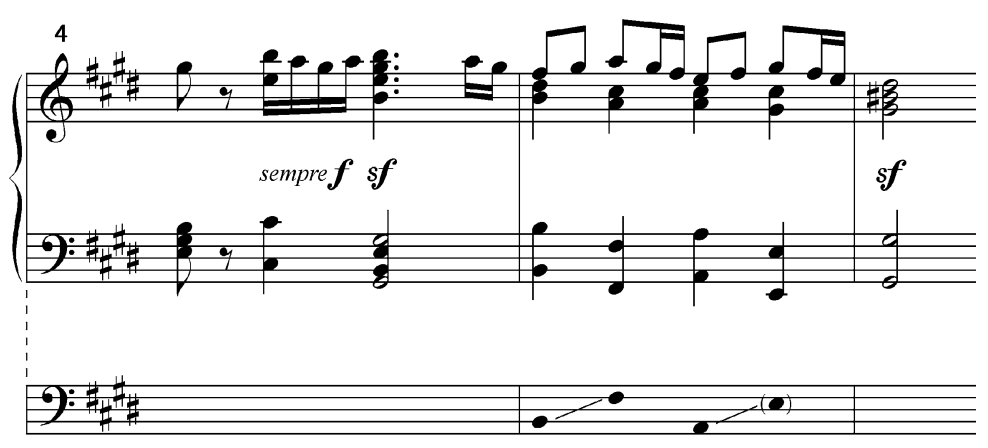

Beispiel 6: Felix Mendelssohn-Bartholdy, Sonate für Klavier op. 7, E-Dur, Finalsatz, T. 4-6

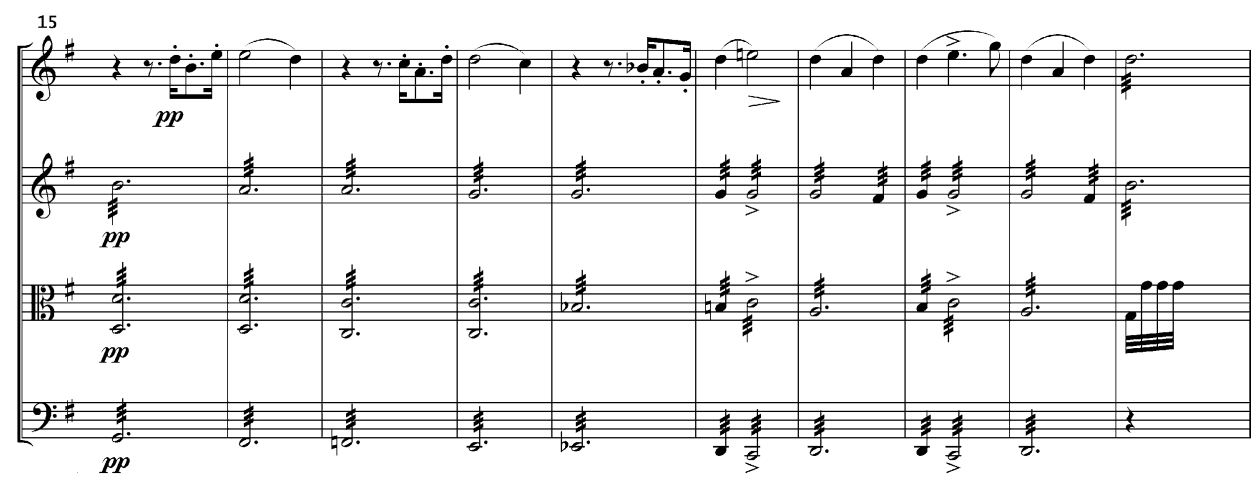

Beispiel 7: Franz Schubert, Streichquartett Nr. 15 G-Dur, D 887,1. Satz, T. 15-24

erinnert. ${ }^{6}$ Er erscheint hier durch den musikalischen Gegensatz der Anfangstakte bewusst vorbereitet und wirkt geradewegs übersteigert in seinem Affekt.

John Leigh bezeichnete diese Takte in einem Gespräch einmal als ıLamento in Dur . Diese Beschreibung bringt das Moment des Außergewöhnlichen der musikalischen Situation in Einklang mit der satztechnischen Ebene, deren Charakteristikum ebenfalls das Abweichen vom `Normalen ist. Und das in mehrfacher Hinsicht: Die chromatische Abwärtsbewegung der Violoncello-Stimme verläuft im Rahmen einer Quarte, überschreitet den Rahmen jedoch zweimal in den Takten 20 und 22. Diese Überschreitungen gehen einher mit einem kurzen Anschwellen und Wiederabflachen der Dynamik. Mit dem Ganztonschritt $d-c$ geht das Modell vom Chromatischen ins Diatonische über. Auch harmonisch fallen die Überschreitungen aus dem Rahmen. Zwischen dem DominantQuartsextakkord und seiner Auflösung in den Grundakkord der V. Stufe erklingt die IV. Stufe. Durch diese Überhöhung wird die folgerichtige Auflösung von 6-4 zu 5-3 hin-

6 Tatsächlich entstand Schuberts letztes Streichquartett parallel zu Beethovens Streichquartett cisMoll, op. 131, vgl. Dürr/Feil 1991, 253. 
ausgezögert. Alle übrigen Harmonien stellen Dur-Akkorde dar, wechselweise in Grundstellung und in erster Umkehrung. Durch die Chromatik der Bassstimme moduliert das Modell rasant, mit jeder weiteren zweitaktigen Sequenzierung werden schnell neue harmonische Regionen erreicht. Ausgehend von dem Quintanstieg G-Dur und D-Dur sind bereits in den Takten 17/18 die Harmonien F-Dur und C-Dur erreicht, im darauffolgenden Takt 19 erklingt Es-Dur. Dem Modell folgend müsste in Takt 20 B-Dur erklingen. Stattdessen vollzieht Schubert hier den Ausstieg aus dem Modell mit der für ihn typischen mediantischen Beziehung Es-Dur/G-Dur.?

Die Takte, in denen Sextakkorde auftreten, enthalten in der ersten Violinstimme weiterhin das kontrapunktische Phänomen der sseptima syncopataı. Vor allem für abwärts geführte Sequenzierungen innerhalb von Fauxbourdon-Sätzen ist diese Synkopendissonanz typisch. Allerdings erinnert die satztechnische Situation mit ihrem Wechsel von Grund- und Sextakkorden an die - wesentlich häufiger in Aufwärtsbewegung anzutreffende - 5-6-Consecutive. Die harmonische Reduktion in der Beispiel 8 verdeutlicht die Vereinigung von 5-6-Consecutive und 7-6-Consecutive in diesem Modell.

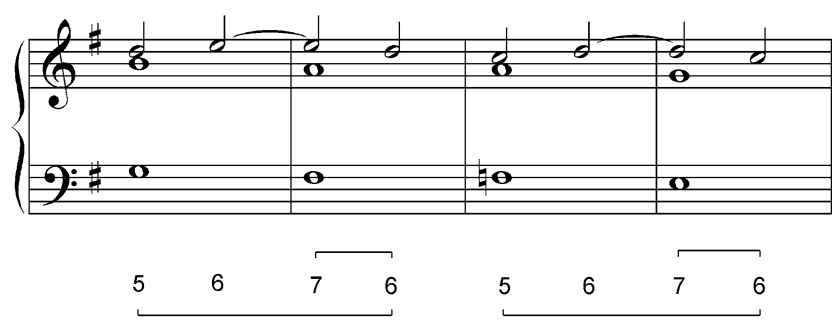

Beispiel 8: Chromatischer Fauxbourdon mit Vereinigung von 5-6-Consecutive und 7-6-Consecutive

Zu einem weiteren Beispiel aus der Romantik: Chopins Etüde op. 10, Nr. 12 c-Moll (Beispiel 9) trägt den Namen »Revolutionsetüde«, was auf ihren nervös-aufrührerischen Charakter zurückzuführen ist, der vor allem durch die unruhigen Sechzehntelläufe in der linken Hand erzeugt wird. Das Stück erreicht den Gipfel seiner Dramaturgie während einer kurzen Sequenzierung, deren harmonische Grundlage ebenfalls fallende Quintanstiege bilden. Das Beispiel zeigt, welche grundverschiedenen musikalischen Inhalte ein und dasselbe satztechnische Gerüst annehmen kann.

Die Personalstile Frédéric Chopins und Robert Schumanns weisen viele Gemeinsamkeiten auf, insbesondere was den Umgang mit Modellen betrifft. Ein Beispiel fallender Quintanstiege findet sich bei Schumann im Mittelteil des dritten Stücks aus den Drei Romanzen op. 28 (Beispiel 10). Hier wird ein eher freundlich daherkommender Abschnitt durch eine ungestüm wirkende chromatische Passage unterbrochen, deren Satztechnik auf den ersten Blick im Vergleich zu der andernorts ausgefeilten Kontrapunktik Schumanns geradezu roh und ungeschliffen wirkt. Dabei beinhaltet die Passage nichts

7 Die Quinte im Bass der Harmonie G-Dur im Takt 20 nimmt allerdings sogleich die Bedeutung eines Dominantquartsextakkords ein, wie oben beschrieben. 

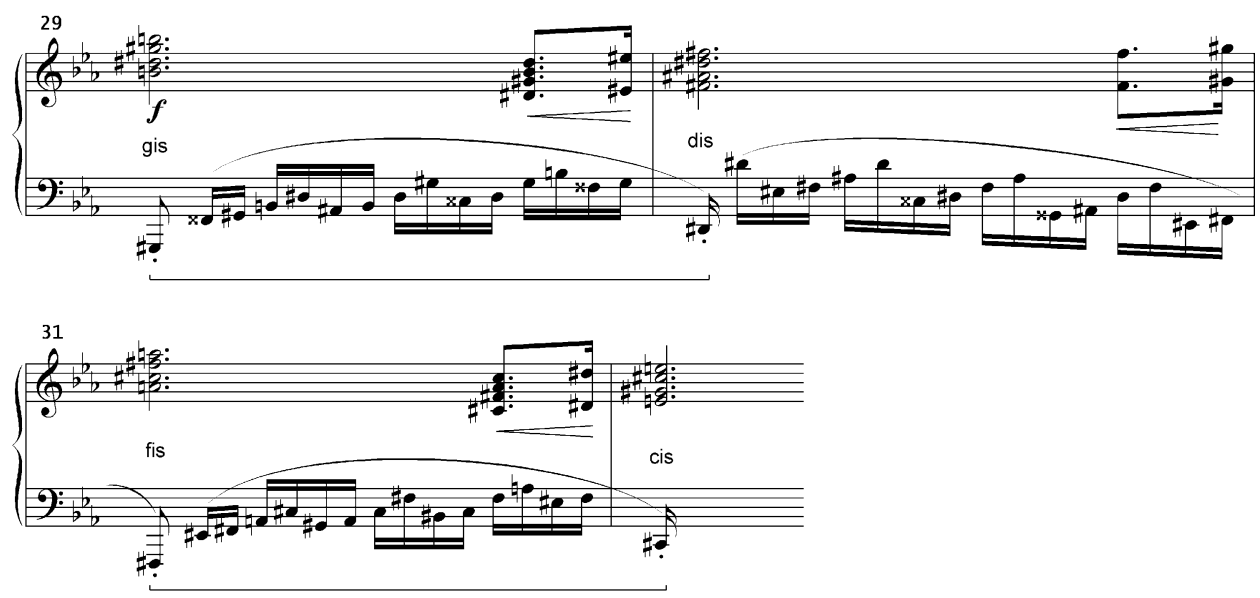

Beispiel 9: Frédéric Chopin, Etüde op. 10, Nr. 12 c-Moll, T. 29-32

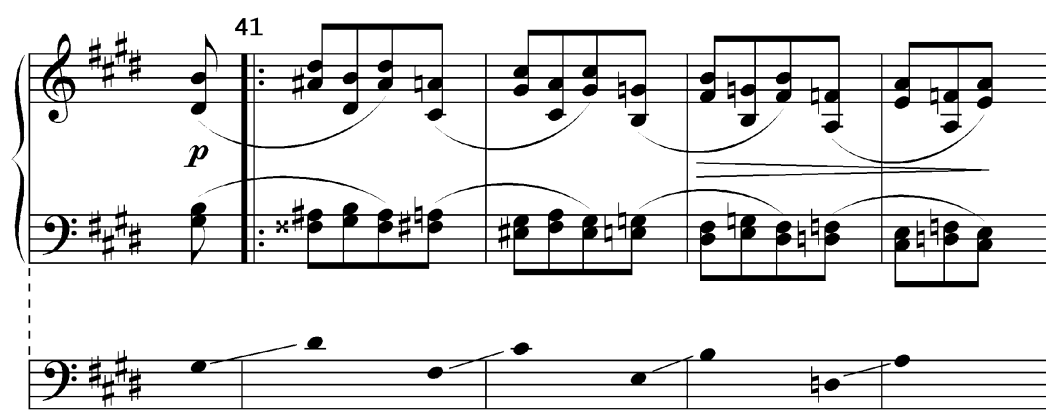

Beispiel 10: Robert Schumann, Drei Romanzen op. 28, Nr. 3, T. 41-448

anderes als den Modulationsweg über absteigende Sequenzierungen, deren Fundamentaltöne zwei um einen Ganzton nach unten versetzte aufsteigende Quinten bilden.

Wie Chopin verwendet auch Schumann für die Ausgangspunkte der Sequenzen Mollakkorde in Grundstellung, die nachfolgenden Dominanten erscheinen bei ihm jedoch als Durakkorde in der ersten Umkehrung. Auf diese Weise entstehen zwei chromatisch abwärtsgerichtete Linien: Eine in der Bassstimme, eine weitere als deren Austerzung in der Tenorstimme bzw. oktaviert wechselweise verteilt auf beide Oberstimmen. Typisch für Schumann - und dies im Unterschied zu Chopin - ist des Weiteren die Idee der Auskomponierung der einzelnen harmonischen Regionen, die hier stufenweise abwärts geführt werden. Auf jeder Stufe erfolgt ein Hin- und Herpendeln zwischen dem Ausgangsakkord

8 Man vergleiche diese Stelle mit den Takten 317-321 gegen Ende des Stücks. Interessant ist dort Schumanns neue Anordnung der Bindebögen, wobei die Töne des Sequenzmodells sich nicht verändern. 

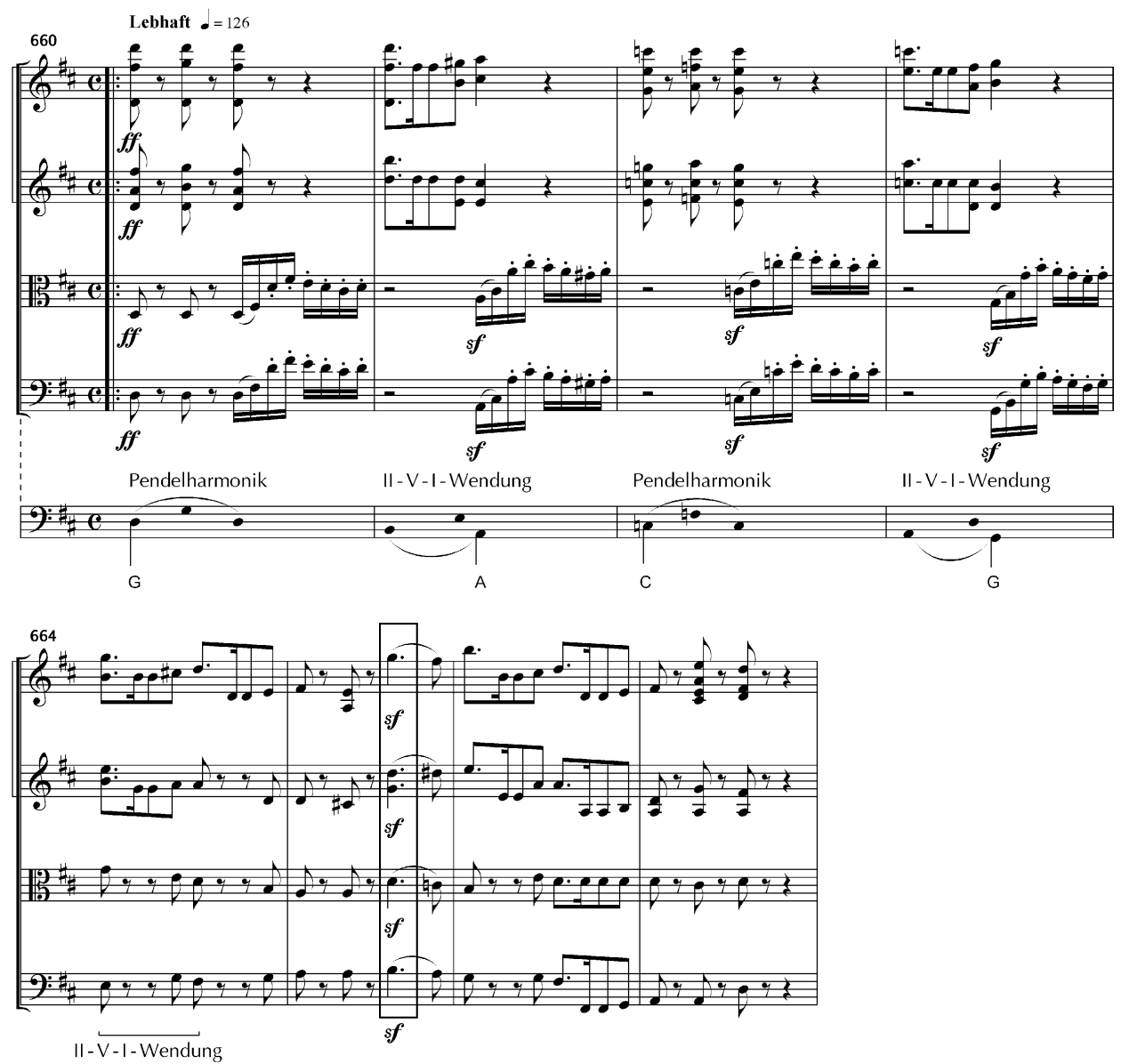

Beispiel 11: Robert Schumann, 4. Sinfonie op. 120 d-Moll, Beginn des 5. Satzes (T. 660-667), Streicher

und seiner Oberquinte, das das Wandern durch die harmonischen Regionen kurzzeitig verzögert. Es handelt sich also um ein durch Pendelharmonik prolongiertes Modell fallender Quintanstiege.

Noch deutlicher wird dieses Charakteristikum Schumannscher Modellbehandlung an den Anfangstakten aus dem 5. Satz seiner 4. Sinfonie (Beispiel 11). Dass die IV. Stufe hier eine besondere harmonische Rolle spielt, offenbaren die Takte 1 und 3. Sie beinhalten jeweils ein kurzes Pendeln zwischen I. Stufe und IV. Stufe, die den Platz der üblicherweise auftretenden V. Stufe einnimmt. Die Takte 3 und 4 sequenzieren die Takte 1 und 2 einen Ganzton tiefer, zusammen bilden sie den Vordersatz eines achttaktigen Satzes. Das erste Sequenzglied beginnt mit der Tonika D-Dur und endet mit einem Halbschluss in A-Dur. Den Ausgangs- und den Endpunkt der zweiten Sequenzierung bilden die Har- 
monien C-Dur und G-Dur. Mithilfe dieser fallenden Quintanstiege, die im Sinne einer Hintergrundreduktion aufzufassen sind, erreicht Schumann am Ende des Vordersatzes wiederum die IV. Stufe. Von ihr aus wird mit der typischen II-V-I-Wendung, die bereits zweimal zuvor in den Takten 2 und 4 erscheint, der Nachsatz eingeleitet. Noch einmal kehrt die Subdominante wieder, erneut an einem Punkt, welcher deren Bedeutung explizit hervorkehrt. Im Takt 665 (dritte Zählzeit) erscheint innerhalb einer trugschlüssigen Wendung anstelle der VI. Stufe (h-Moll) ein G-Dur-Sextakkord. Mit nur einem geänderten Ton ( $g$ statt $h$ ) ändert sich die Wirkung dieser Stelle komplett, was im Notentext mit einem $s f$ unterstrichen wird. Wiederum erscheint das Modell, als Eröffnungstopos genutzt, prolongiert: Die harmonischen Eckpunkte sind in beiden Sequenzierungen auskomponiert durch Pendelharmonik zwischen I. und IV. Stufe sowie eine anschließende abkadenzierende II-V-I-Wendung, die sich auf die jeweils folgenden Oberquinttonarten (A-Dur bzw. G-Dur) beziehen.

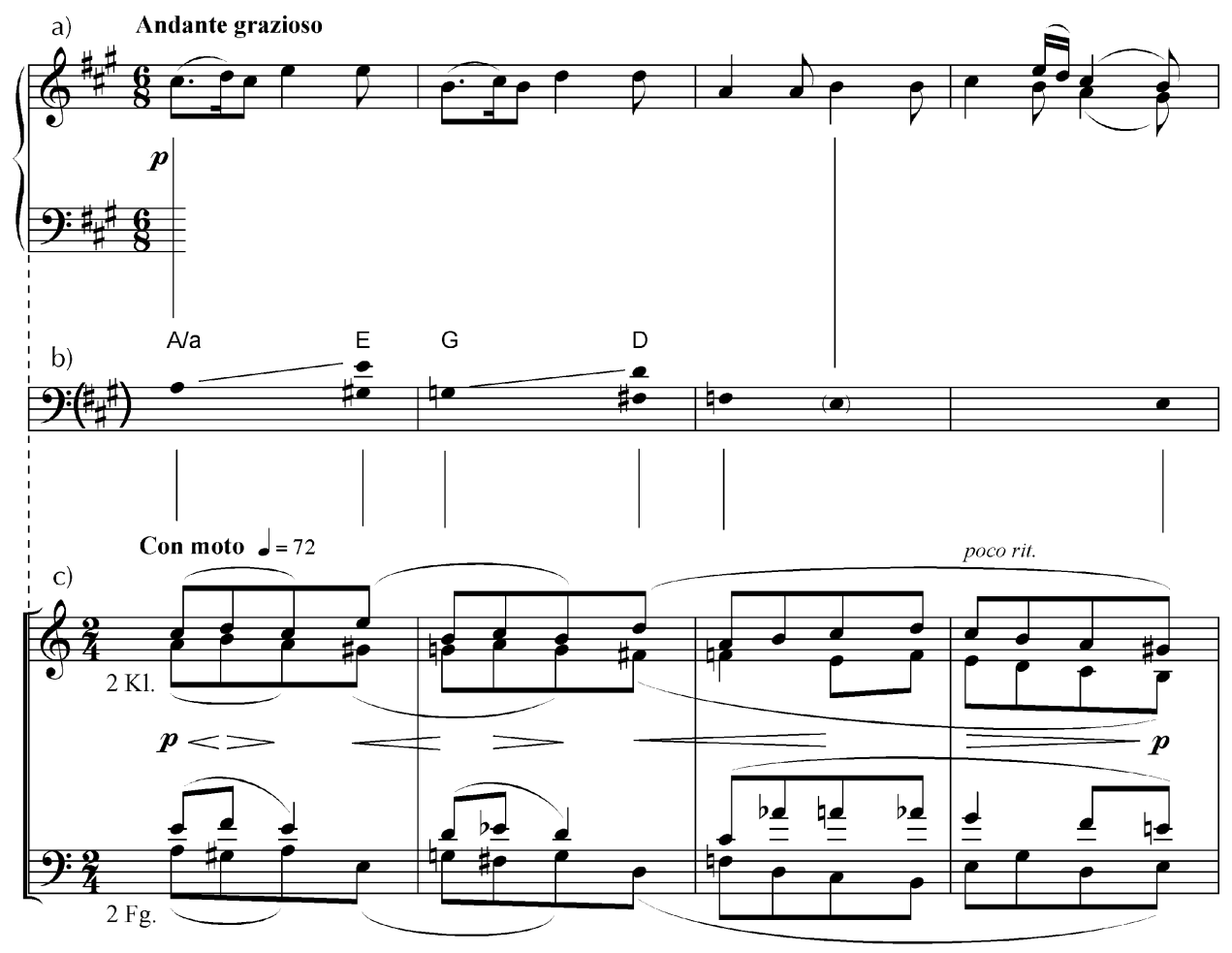

Beispiel 12:

a) Wolfgang Amadeus Mozart, Klaviersonate KV 331, A-Dur, Eröffnungstakte;

b) potentielle Harmonisierung durch einen chromatischen Lamento-Bass;

c) tatsächliche Harmonisierung in Max Reger, Variationen und Fuge über ein Thema von Mozart op. 132, Beginn der Var. III, (Holzbläser, Klarinetten nicht transponierend) 
Auch Mozarts berühmtes Thema aus der Klaviersonate KV 331 beginnt mit abwärts gerichteten Sequenzierungen und liefert damit die Grundlage für eine - im Sinne der Charaktervariation des 19. Jahrhunderts - neue Harmonisierung. Diese Möglichkeit nutzt Max Reger in seinen Mozart-Variationen op. 132. Die fallenden Quintanstiege erscheinen bei ihm wiederum als Eröffnungsmodell, außerdem bezeichnenderweise innerhalb einer Minore-Variation, was seine Verwandtschaft mit dem (chromatischen) Lamento verdeutlicht.

Regers Modell (Beispiel 12c) weist dabei erstaunliche Parallelen zu Schumanns fallenden Quintanstiegen in der Romanze op. 28, Nr. 3 auf (vgl. Beispiel 10). Auch bei Reger wird der erste Grundakkord eines jeden Sequenzgliedes (a-Moll, G-Dur, F-Dur) auskomponiert. Das Mittel der Prolongation ist in den ersten beiden Takten ebenfalls eine Pendelharmonik, die sich allerdings von Schumanns Variante unterscheidet. Bei Schumann entsteht das Pendeln durch die Wiederholung des Wechsels zwischen dem Start- und dem Zielakkord jedes Sequenzgliedes (also zwischen Moll-Grundakkord und Dur-Sextakkord). Bei Reger hingegen erscheint der Zielakkord ebenfalls in Grundstellung, eine weitere eingeschobene V. Stufe (an zweiter Stelle jedes Sequenzgliedes) erfolgt mit einem dominantischen verminderten Septakkord.

\section{Coda - Crossover}

Bis in die (tonale) Musik der Gegenwart hinein lässt sich die Verwendung fallender Quintanstiege als satztechnisches Modell verfolgen. Insbesondere in Rock- und Poptiteln erscheinen häufig immer wiederkehrende Harmoniefolgen, die sich - betrachtet durch die Brille der «klassischen « Musiktheorie - auf diese Weise klassifizieren lassen. Einen Stammplatz nehmen fallende Quintanstiege dabei in den Bridge-Teilen ein, in den »neuen Strophen « also. In Analogie zu den oben aufgeführten Beispielen der `E-Musikı stehen sie auch hier für etwas Besonderes, aus dem bisherigen musikalischen Umfeld Ausbrechendes (Beispiel 13). Beispiele sind die Titel Du erinnerst mich an Liebe (Ich + Ich) oder auch Don't Speak (No Doubt). Aber auch andernorts sind fallende Quintanstiege in Rock- und Poptiteln anzutreffen. So bildet dieses Modell die harmonische Grundlage des gesamten Songs I'm Outta Love von Anastacia (in der Beispiel 13 dargestellt anhand des Refrains). Gleiches gilt für den Titel Take Me Home von Phil Collins.

»In Topoi wird Rezeptionsgeschichte greifbar. «" Am Umgang verschiedener Stilepochen und - wesentlich differenzierter - einzelner Komponisten mit musikalischen Topoi lässt sich ein Stück Musikgeschichte (Kompositionsgeschichte, Geschichte der Musiktheorie) anschaulich darstellen. ${ }^{10}$ So auch anhand fallender Quintanstiege, die vorangehend als

9 Jeßulat 2001, 118.

10 Mit dieser Lehrtradition, die vornehmlich auf die Städte Basel, Berlin und Hamburg zurückgeht, ist eine Kritik an ahistorischen Systematiken, insbesondere an der Funktionstheorie, verbunden, siehe hierzu Aerts 2007. 
Ich + Ich, Du erinnerst mich an Liebe, Bridge
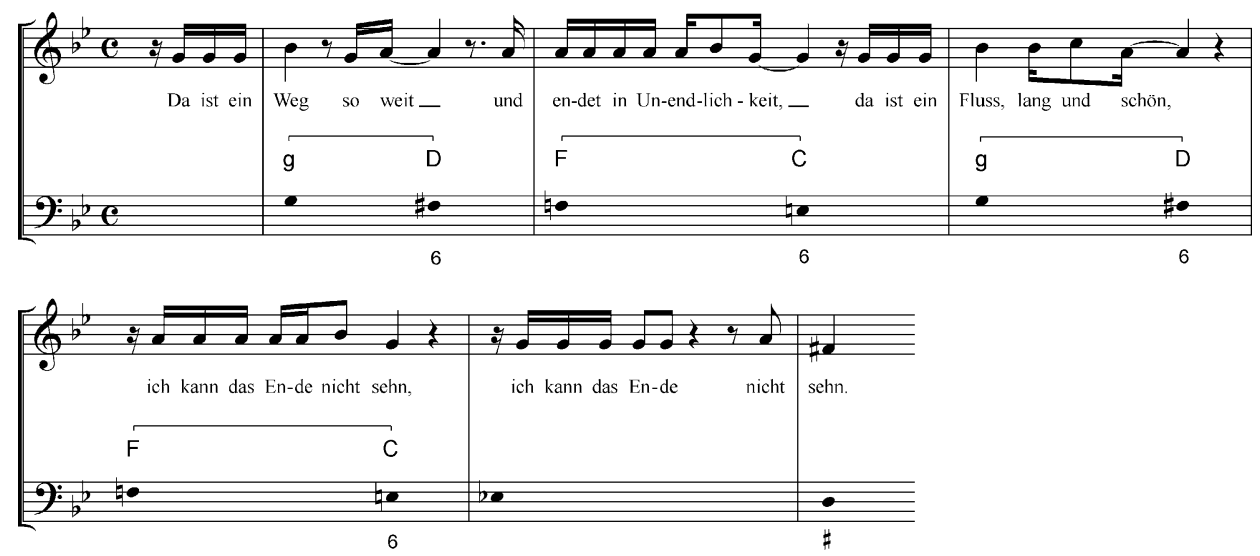

No Doubt, Don't Speak, Bridge

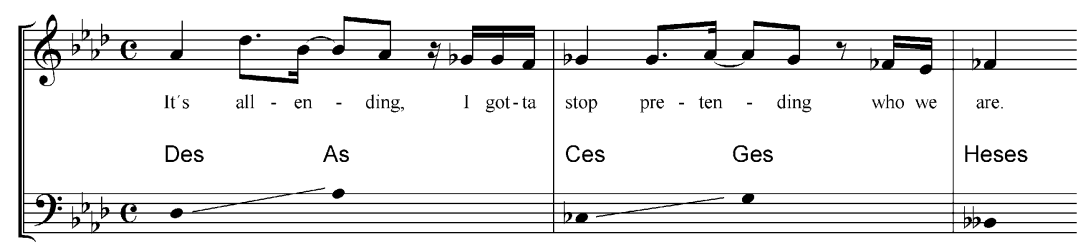

Anastacia, I'm Outta Love, Refrain

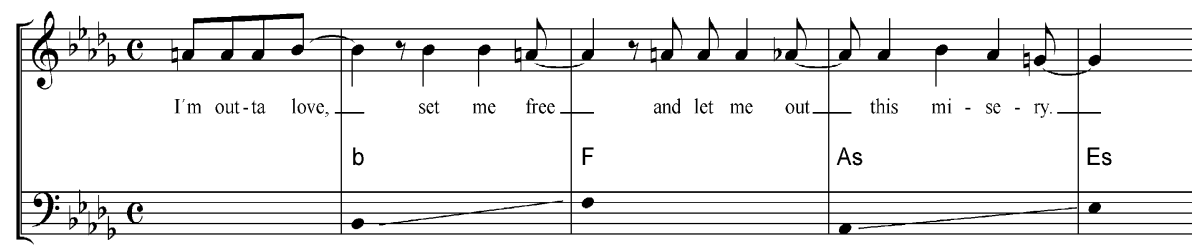

Phil Collins, Take Me Home

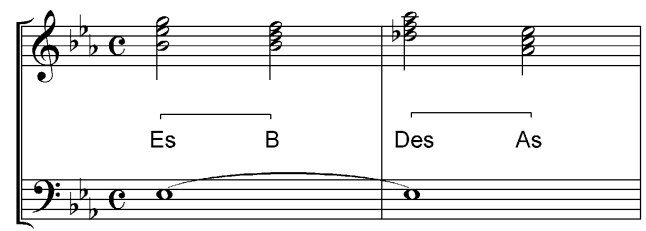

fallende Quintanstiege über Orgelpunkt als harmonisches , Patterns

Beispiel 13: Fallende Quintanstiege in einigen Rock-/Popsongs 
eigenständiger Topos definiert worden sind, abgeleitet vom gemischt chromatisch-diatonischen Lamento und in enger Verwandtschaft zu aufsteigenden Quintfällen stehend. Die harmonische Ausgestaltung abwärts gerichteter Sequenzierungen erfolgte im Laufe der Jahrhunderte in unterschiedlichen musikalischen Situationen auf vielfältige Art und Weise. Dabei bildete sich nach und nach ein breites Spektrum an semantischen Bedeutungen heraus, die fallende Quintanstiege annehmen konnten. Stets lassen sich diese aber auf einen satztechnischen resp. harmonisch-kontrapunktischen Kern reduzieren. In bemerkenswerter Weise ist ein fortdauernder Umgang mit historischen Satzmodellen in der sogenannten Gebrauchs- oder Unterhaltungsmusik zu beobachten. Durch eine permanente Individualisierung von Topoi wird die Modellpraxis am Leben gehalten, ohne dass sie als bloßer kompositorischer Archetypus fungiert.

\section{Literatur}

Aerts, Hans (2007) » Modell und „Topos` in der deutschsprachigen Musik seit Riemann«, ZCMTH 4/1-2, 143-158.

Dürr, Walther / Arnold Feil (1991), Reclams Musikführer. Franz Schubert, Stuttgart: Reclam.

Fladt, Hartmut (2005a), »Modell und Topos im musiktheoretischen Diskurs. Systematiken/Anregungen«, Musiktheorie 4/20, 343-369.

— (2005b), »Satztechnische Topoi«, ZGMTH 1/2, 189-196.

Froebe, Folker (2007a), »Historisches Panoptikum der Satzmodelle«, ZGMTH 4/1-2, 185-195.

(2007b), „Satzmodelle des `Contrapunto alla mente` und ihre Bedeutung für den Stilwandel um 1600«, ZCMTH 4/1-2, 13-56.

— (i. V), »Sequenz und Imitation - Satzmodelle `Note gegen Note orie des 18. Jahrhunderts", in: Musiktheorie und Vermittlung - Tagungsbericht des VI. Kongresses der Gesellschaft für Musiktheorie (GMTH), hg. von Klaus Heiwolt, Hildesheim u. a.: Olms.

Jeßulat, Ariane (2001), Die Frage als musikalischer Topos. Studien zur Motivbildung in der Musik des 19. Jahrhunderts, Diss. Berlin (= Berliner Musikstudien 21), Sinzig: Studio.

Muffat, Georg (1699), Regulae concentuum partiturae, Ms., hg. von Hellmut Federhofer als: An Essay on Thoroughbass (= Musicological Studies and Documents 4), Dallas: American Institut of Musicology 1961.

Zacconi, Ludovico (1622), Prattica di Musica, 2 Bde., Venedig: Carampello 1596 und Vincenti 1622, Reprint Hildesheim u. a.: Olms 1982. 